\title{
EL DESPERDICIO ALIMENTARIO EN COMEDORES ESCOLARES Y SU PERCEPCIÓN ENTRE EL ALUMNADO Y LAS FAMILIAS
}

\author{
$\underline{\text { María Ángeles Fernández-Zamudio }}^{\mathrm{a}^{*} \text { y Tatiana Pina }}{ }^{\mathrm{b}}$
}

a Instituto Valenciano de Investigaciones Agrarias. Centro para el Desarrollo de la Agricultura Sostenible (Moncada-Valencia, fernandez_marzam@gva.es). ${ }^{b}$ Universitat de València. Facultad de Magisterio (Valencia, tatiana.pina@uv.es).

\begin{abstract}
Resumen
El desperdicio alimentario (DA) es un problema global que ocasiona importantes impactos económicos, sociales y ambientales, y un gran desconocido por parte de la sociedad, que ignora las consecuencias reales y su alcance. Individualmente la ciudadanía percibe que es un problema ajeno, del que no se siente ni partícipe ni responsable.

En el presente trabajo se muestran los resultados de un estudio realizado en siete colegios de la Comunitat Valenciana. Por un lado, se ha cuantificado cuánta comida se desperdiciaba en los comedores escolares, para lo que se midió el DA durante dos semanas ( $\mathrm{n}=4.241$ servicios). Y por otro lado, se quería confirmar el grado de conocimiento y la percepción del DA que tenía parte de la comunidad escolar a través de un sondeo al alumnado $(n=363)$ y a las familias $(n=438)$. Los datos obtenidos han permitido ahondar en esta cuestión y ver la relación de ciertos comportamientos en función de distintas variables sociodemográficas. Sensibilizar y concienciar sobre el problema del DA en las escuelas es una manera efectiva de introducir buenos hábitos en los hogares, incentivando el consumo responsable, algo que se traspasa del colegio a las familias y con ello a la ciudadanía en su globalidad.
\end{abstract}

Palabras clave: gestión comida, comedores escolares, comportamiento alimentario, educación ambiental, consumo responsable.

\section{Introducción y objetivos}

En el estudio encargado por la FAO en 2011 sobre las pérdidas y el desperdicio alimentario global se señala que de media un tercio de todos los alimentos producidos en el mundo se pierden o se desperdician (Gustavsson et al, 2011), lo que asciende a 1.300 millones de toneladas cada año. Según el Ministerio de Agricultura español, España es el séptimo país que más desperdicia en Europa, con una cifra estimada de 7,7 millones de toneladas de residuos de alimento al año, lo que equivale a una media per cápita de $170 \mathrm{~kg}$ (MAPA, 2019). Dada la gran envergadura de las cifras del desperdicio alimentario (DA), las grandes organizaciones mundiales están incluyendo desde hace años este problema en sus principales programas de trabajo. Es el caso de la Unión Europea que ha decidido incluir la reducción del DA en la Agenda de la Economía Circular, y con ello tratar de cumplir los niveles que se marcan desde la Organización de las Naciones Unidas (ONU) en los Objetivos de Desarrollo Sostenible (ODS). Concretamente la meta 12.3 fija una reducción del 50\% del total de residuos que se generan en la cadena para el año 2030. Cumplir esta meta sin duda también contribuiría al logro de la meta 2.4 que pretende asegurar la sostenibilidad de los sistemas de producción de alimentos.

Para tratar de concienciar a la ciudadanía de la gravedad de este problema global e implicarla en un cambio de hábitos dirigido a conseguir un consumo más responsable y sostenible, se cree imprescindible intervenir en los primeros niveles de su formación, esto es, en las etapas de Educación Infantil y Primaria. Además, como lugares de restauración colectiva que son, los comedores escolares generan cada año un importante volumen de DA. En realidad, trabajar para la visibilización, sensibilización y educación del problema del DA es una estrategia fundamental para combatirlo y el abordaje de este tema en los centros escolares supone una gran oportunidad para provocar un cambio de hábitos de consumo e influir así en el estilo de vida de niños y niñas, docentes, personal laboral y familias.

El objetivo de este trabajo es analizar desde una doble perspectiva el problema del DA en siete centros de Educación Infantil y Primaria (CEIPs) de la Comunitat Valenciana. En primer lugar midiendo el DA que se genera en sus comedores, y en segundo lugar sondeando entre la comunidad escolar cuál era la percepción y nivel de conocimiento que tenían tanto el alumnado como sus familiares.

\section{Metodología}

Los siete CEIPs que se eligieron están ubicados en el sur de la provincia de Valencia y todos tenían comedores que eran servidos a través de un catering externo. La medición se realizó durante dos semanas consecutivas en los meses de mayo y junio de 2019. En el comedor escolar únicamente se cuantificó el desperdicio originado en el plato siguiendo las directrices del estudio piloto realizado por el entonces 
Ministerio de Agricultura, Pesca y Alimentación (Agustín y Derqui, 2016): antes del servicio se medía el peso medio de las raciones, y se pesaba la fracción comestible, identificando el volumen de las partes no comestibles en el caso de haberlas (huesos, espinas, etc.). La cuantificación del desperdicio global generado se hizo siguiendo el método de pesado agregado selectivo, con el que se simplifica y acelera la recogida de datos, ya que se realizaba el pesado total de los desperdicios de cada turno de comida, se separaban los restos de comida diferenciando según el tipo plato, y posteriormente se calculaba el promedio por comensal. Durante el período de estudio se midieron un total de 4.241 menús servidos en los siete CEIPs.

El sondeo se realizó a alumnado de edades comprendidas entre los 6 y los 12 años (n=363 respuestas), gracias al soporte prestado por el profesorado. En aquellos casos en los que el alumnado tenía la edad suficiente como para usar los ordenadores, eran ellos mismos quienes introducían los datos en el cuestionario distribuido de forma virtual; en el caso del alumnado de menor edad, los cuestionarios se distribuyeron en papel. Para recoger las respuestas de los familiares ( $\mathrm{n}=438$ respuestas) se aprovecharon las visitas a tutorías habituales al final de curso, y todos los cuestionarios se rellenaron en las dependencias del colegio y en formato papel.

\section{Resultados}

\subsection{Cuantificación del desperdicio en los comedores}

De la revisión de los menús que se consumieron en los días del estudio en los siete colegios, se deduce que siguen planes de nutrición con pautas similares. Al medir el DA generado durante dos semanas se cubre la variedad de platos con los que suelen alternar, por lo que los resultados podrían ser equivalentes en otros momentos del curso académico. Las raciones se consideran equilibradas, en volumen y tipología de alimentos, siendo habitual ofrecer los alimentos como carnes o pescados sin pieles, ni espinas ni huesos, por lo que en la mayoría de los platos la fracción no comestible era inexistente.

Según el centro, la comida se servía en bandeja o plato, si bien el tamaño de las raciones era equivalente entre alumnado del mismo turno, y ligeramente menores en el turno de Infantil respecto al de Primaria. En la mayoría de los comedores había posibilidad de repetir. En los centros C2 y C6, la comida se servía en un recipiente central en la mesa, siendo repartida por uno de los comensales; en estos casos se considera que las raciones podrían ser más individualizadas, aunque en todos los casos había monitores/as supervisando que se repartiera y se ingiriera una ración mínima.

Tras valorar la porción de comida servida y desperdiciada se obtenía el desperdicio en \% de cada menú. Este valor resultó ser de media para los siete CEIPs y todos los menús servidos de un $25 \%$. Valorando por tipología de plato, el entrante, que suele ser ensalada, es lo que porcentualmente más se desperdicia, casi el $34 \%$, y el segundo plato, el que menos, con aproximadamente el $21 \%$. Aunque también se midió el desperdicio generado de postre y el pan, la disparidad de medidas hace que no se incluya en la síntesis de resultados mostrada en el Cuadro 1. Entre tipos de alimentos la diferencia fue grande, los potajes de legumbres, cremas de verdura, o pescados solían ser más rechazadas, mientras que croquetas, patatas fritas, o pastas tenían menos desperdicio.

Cuadro 1. Desperdicio alimentario cuantificado en los comedores de siete centros educativos en la Comunitat Valenciana

\begin{tabular}{|c|c|c|c|c|c|c|}
\hline Educativo & $\begin{array}{c}\text { Comida servida** } \\
\text { (g/pers.) }\end{array}$ & $\begin{array}{c}\text { Desperdicio } \\
\text { generado** } \\
\text { (g/pers.) }\end{array}$ & $\begin{array}{c}\text { Desperdicio total } \\
\text { en el menú } \\
\%\end{array}$ & $\begin{array}{c}\text { DA en Entrante } \\
\%\end{array}$ & $\begin{array}{c}\text { DA en Plato } 1^{\circ} \\
\%\end{array}$ & $\begin{array}{c}\text { DA en Plato } 2^{\circ} \\
\%\end{array}$ \\
\hline $\mathrm{C} 1$ & 421,87 & 156,09 & 37,0 & 74,2 & 43,6 & 30,0 \\
\hline $\mathrm{C} 2$ & 423,73 & 87,10 & 20,6 & 11,8 & 24,9 & 15,6 \\
\hline $\mathrm{C} 3$ & 458,87 & 127,73 & 27,8 & 7,9 & 38,3 & 23,7 \\
\hline $\mathrm{C} 4$ & 453,24 & 55,55 & 12,3 & 17,6 & 8,4 & 9,2 \\
\hline $\mathrm{C} 5$ & 428,44 & 133,22 & 31,1 & 60,2 & 30,0 & 17,6 \\
\hline C6 & 474,82 & 99,70 & 21,0 & 48,7 & 18,1 & 22,0 \\
\hline $\mathrm{C} 7$ & 553,56 & 134,73 & 24,3 & 16,4 & 24,0 & 27,2 \\
\hline Media & 459,22 & 113,44 & 24,9 & 33,8 & 26,8 & 20,8 \\
\hline
\end{tabular}

* Los Colegios de C1 a C5 están ubicados en municipios pequeños y de carácter rural. El C6 está en un municipio mediano de carácter urbano en la costa, y el C7 también está en un municipio mediano en el interior donde predomina la actividad industrial y rural.

** Se refieren valores medio, contemplando todos los turnos y días de medición.

Considerando una media para el conjunto de turnos, CEIPs y días de medición, el DA del menú expresado en gramos por comensal resulta de 113,44 g, lo que supone algo más que en el estudio de Agustín y Derqui (2016) que determinaron entre 60 y 100 g de DA por estudiante y día para una muestra menor de CEIPs. En la bibliografía los datos oscilan bastante, y lo habitual es que cada uno utilice un protocolo y planteamientos distintos en la forma de medir, lo que dificulta la comparativa.

\subsection{Sondeo al alumnado}


El cuestionario del alumnado tenía un primer bloque de cuestiones en el que se pedían ciertas valoraciones, sobre si tiraban alimentos durante el almuerzo (descanso de media mañana) y en la comida principal en el comedor, el motivo del por qué desperdiciaban alimentos y qué consecuencias veían en ese hecho. El sondeo se completaba con preguntas relacionadas con aprendizajes que suelen adquirirse en familia. Respecto al almuerzo, lo más habitual es que indicaran que no lo tiraban porque se lo comían todo (49\% del alumnado), o si les sobraba se lo llevaban a casa (27\%), o bien que lo tiraban puntualmente (23\%). Solo un $1 \%$ de los 359 niños/as que respondieron, indicaron que lo tiraban todos los días. Los motivos de porqué tiraban el almuerzo se recogen en el Gráfico 1, siendo lo más habitual la respuesta "No tengo más hambre". Respecto al comedor, tampoco reconocen que tiran alimentos (un 50\% dice no tirar nunca), y cuando lo hacen (el 33\% dice tirarlos algunas veces y un 17\% siempre) las respuestas más frecuentes fueron "no me gustan los sabores de algunos alimentos", o bien "porque no tengo hambre".
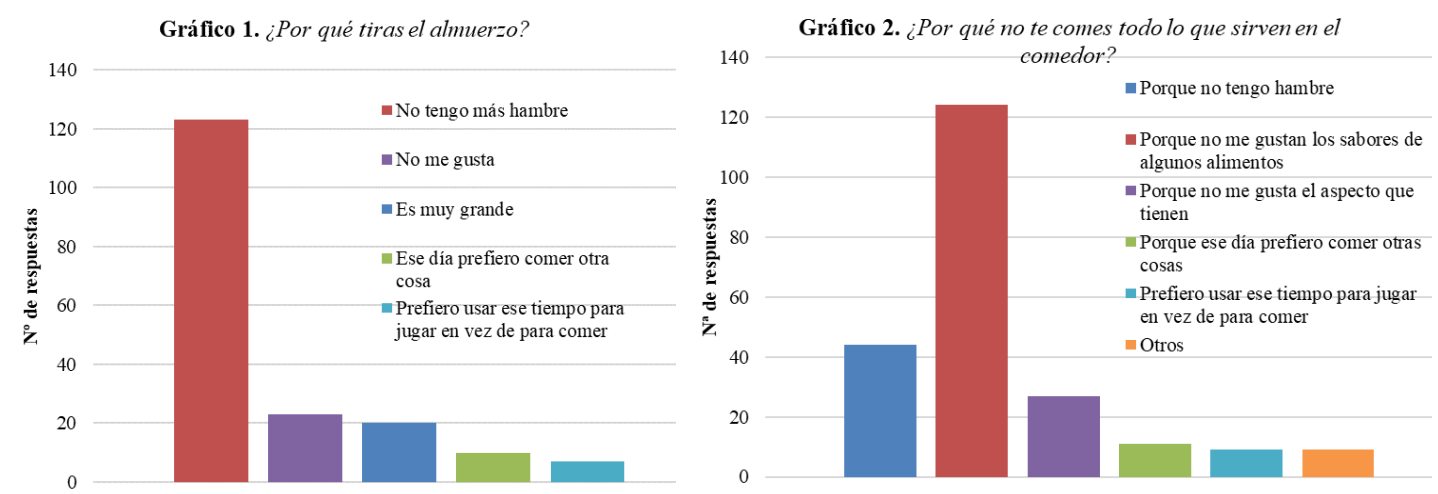

Cuando se les preguntaba por las consecuencias de ese desperdicio, un 25\% atribuyó aspectos sociales ("Que gente se quede sin comida"), en torno al 24\% argumentó aspectos económicos ("Tirar dinero"), y solo un 9\% mencionó aspectos ambientales ("Que hace daño al medio ambiente"). En torno al 7\% explicó que era una combinación de las tres dimensiones, ambiental, social y económica. El resto de alumnado dijo desconocerlas o bien argumentó otras consecuencias difícilmente catalogables en esas dimensiones (un $35 \%$ ). Por otra parte, se les preguntó si en casa sus familiares les hablaban alguna vez de la necesidad de realizar ciertas prácticas, como: ayudar a personas necesitadas (un 8,8\% del alumnado indicó que no se hablaba en casa); pedir o comprar solo la comida que van a comer (el 15,17\% dijo que no se hablaba); cómo conservar mejor los alimentos (el 21,5\% dijo que no se hablaba) o la necesidad de no tirar comida (el 26\% dijo que no se hablaba), de ahorrar luz (no se hablaba en el 11,8\% de los casos) o de no malgastar el agua (el $23 \%$ dijo que no se hablaba). Y finalmente, el $15,7 \%$ dijo que no se mencionaba en casa el valor que tiene lo que comemos. Se deduce, por lo tanto, que en cuanto a este grupo de hábitos saludables para las personas y el medio ambiente hay un amplio margen de mejora, y las familias deben asumir esta responsabilidad, que deberá ser compartida y complementada con lo que aprendan en el colegio.

\subsection{Sondeo a las familias}

El cuestionario que fue respondido por las familias $(n=438)$ estaba constituido por diversos bloques que abordaban desde hábitos alimentarios, de compra y cocinado, de generación de residuos y reciclaje, etc. Sin embargo, a causa del reducido espacio del que se dispone en este trabajo, tan solo se muestran las respuestas relacionadas estrictamente con el desperdicio. En el Cuadro 2 se detallan las principales características sociodemográficas de los familiares encuestados para enmarcar mejor dichas respuestas.

Las familias valoraron su desperdicio entre 5 y 10 euros al mes (42\% de las respuestas) de entre un rango que iba desde menos de 5 euros a más de 20 euros, siendo la siguiente respuesta más escogida, menos de 5 euros $(29,5 \%)$. Y cuando se les preguntó qué hacían con los restos o platos de comida que les sobraban el $72 \%$ decía conservarlos con cierta frecuencia o muy frecuentemente y solo un $9,3 \%$ indicaba que los tiraba con la misma frecuencia. Un $62 \%$ indicaba que estos restos los congelaba frente al $66 \%$ que indicaba que nunca o solo a veces, reorganizaba las comidas y hacía nuevos platos con recetas de aprovechamiento. Aquellos ítems en los que se les preguntaba si hablaba con sus hijos e hijas sobre la importancia de no tirar comida, conservar mejor los alimentos, la necesidad de ahorrar agua o usar adecuadamente la luz eléctrica se analizaron mediante una tabla de contingencia comparándose con las respuestas a las mismas preguntas del alumnado. Como resultado se observó que la variable "sujeto" (alumnado y familia) y "respuesta" (Nunca, a veces y frecuentemente) están fuertemente relacionadas en las cuatro preguntas analizadas (Pvalor $<0,001)$ y en todos los casos se observaba que entre el alumnado había menos respuestas de "frecuentemente" y más de "nunca" o "a veces", de lo que se esperaba, mientras que en el caso de las familias, había más "frecuentemente" y menos "nunca o a veces" de lo pronosticado, situación que apunta que en los hogares se debería insistir más en este tipo de cuestiones para que permeen en sus hijos e hijas. 
Cuadro 2. Características sociodemográficas de la muestra

\begin{tabular}{|c|c|c|c|c|c|}
\hline \multicolumn{2}{|c|}{ Categoría sociodemográfica } & \multirow{2}{*}{$\frac{\mathbf{N}^{\mathbf{2}}}{338}$} & \multicolumn{2}{|c|}{ Categoría sociodemográfica } & \multirow{2}{*}{$\frac{\mathbf{N}^{\mathbf{2}}}{0}$} \\
\hline \multirow{3}{*}{ Familiar } & Madre & & \multirow{6}{*}{$\begin{array}{c}\mathrm{N}^{\circ} \\
\text { personas/hogar }\end{array}$} & 1 & \\
\hline & Padre & 82 & & 2 & 21 \\
\hline & No declarado & 18 & & 3 & 103 \\
\hline \multirow{6}{*}{ Edad } & $20-30$ & 6 & & 4 & 252 \\
\hline & $31-40$ & 136 & & 5 o más & 40 \\
\hline & $41-50$ & 188 & & No declarado & 22 \\
\hline & $51-60$ & 11 & \multirow{3}{*}{$\begin{array}{l}\mathrm{N}^{\circ} \text { personas } \\
\text { menores de } 16 \\
\text { años }\end{array}$} & 1 & 140 \\
\hline & $61-70$ & 1 & & 2 & 201 \\
\hline & No declarado & 96 & & 3 & 18 \\
\hline \multirow{7}{*}{ Estudios } & Educación Primaria & 121 & \multirow{7}{*}{ Ingresos Brutos } & Hasta $12.000 €$ & 148 \\
\hline & Educación secundaria & 74 & & 12.001 a $18.000 €$ & 102 \\
\hline & Formación Profesional & 94 & & 18.001 a $24.000 €$ & 52 \\
\hline & Universitarios & 124 & & 24.001 a $36.000 €$ & 38 \\
\hline & Postgrado & 18 & & Más de $36.000 €$ & 18 \\
\hline & \multirow[t]{2}{*}{ No declarado } & 7 & & Hasta $12.000 €$ & 148 \\
\hline & & & & No declarado & 80 \\
\hline
\end{tabular}

\section{Discusión y Conclusiones}

Del estudio realizado en siete CEIPs de la Comunitat Valenciana se han extraído enseñanzas que podrían extrapolarse al conjunto de la comunidad escolar. Respecto al desperdicio generado en los comedores por el alumnado, se observa que a pesar de que las raciones están muy tasadas por las empresas de catering y que al menos en la muestra analizada recibían la comida prácticamente sin partes no comestibles, al final el desperdicio medio del menú puede llegar al $25 \%$ de la cantidad aportada, una cifra que se considera alta. Esta cifra aún es mayor en los alimentos que se dan de entrante (normalmente ensaladas) y mayor en los primeros platos que en los segundos, normalmente debido a las preferencias del alumnado, algo que en gran medida también se marca desde los hogares. Hay prácticas como la de servir de forma centralizada en la propia mesa, de manera que se regule la cantidad de comida que se distribuye, que parecen ser positivas para evitar el DA, aunque por logística esto no se practica en la mayoría de centros. Un buen ambiente de luz, temperatura o ruido en el comedor, y el hecho de que haya personas de confianza sirviendo o vigilando el comedor, suelen ser favorables para reducir el DA. En cualquier caso hay que seguir trabajando con los responsables del comedor y con las empresas que sirven los catering para que se haga una alimentación lo más completa y atractiva para el alumnado, tanto en su aspecto como en palatabilidad. Formar en el aula sobre el valor de los alimentos y que vivencien la comida como un momento de placer, también ayuda a que adquieran buenos hábitos. Respecto a lo que se percibe en las encuestas, la conclusión mayor es que hay que seguir trabajando para que conozcan la importancia que tiene el desperdicio en las tres dimensiones de la sostenibilidad. El trabajo con los centros de infantil y primaria debe ser prioritario, porque la sensibilización y conocimientos que se adquieren en los mismos marcan el comportamiento futuro del alumnado, y desde ellos se puede llegar fácilmente a los hogares y al conjunto de la ciudadanía.

\section{Agradecimientos}

Estudio enmarcado en el "Plan contra el desperdicio alimentario en la Comunitat Valenciana, BonProfit", que cuenta con fondos FEDER. Agradecemos a la comunidad escolar de los centros educativos que intervinieron en el estudio, su tiempo, disposición para facilitar la medición en los comedores, y el acceso al alumnado y familias. También al Consorci de Residus COR-V5 por el soporte prestado.

\section{Bibliografía}

Agustín, A. y Derqui, B. (2016). "Estudio piloto para la medición y reducción del desperdicio de alimentos en comedores escolares: Auditoría y Autoevaluación. Technical report". En Catálogo de Publicaciones de la Administración General del Estado.

Gustavsson, J., Cederberg, C., Sonesson, U. y Meybeck, A. (2011). Global Food Losses and Food WasteExtent, Causes and Prevention. 1st ed. FAO: Gothenburg. ISBN 9780786495566.

MAPA (2019). "Portal más alimento, menos desperdicio. Cifras de las pérdidas y del desperdicio alimentario". Disponible en https://www.menosdesperdicio.es/definiciones-cifras/cifras 